Trauma Berufskrankh 2010 · 12[Suppl 2]:194-198 DOI 10.1007/s10039-010-1612-0

Online publiziert: 3. April 2010

(c) Springer-Verlag 2010

\author{
S. Simmel \\ Abteilung für BG-Rehabilitation, BG-Unfallklinik Murnau, Murnau am Staffelsee
}

\title{
Frührehabilitation nach Polytrauma
}

zungsbedingte Faktoren (Lokalisation, Schweregrad, Anzahl der Verletzungen) $[2,5,7]$ sind wichtige Outcome-Determinanten. Trotzdem können diese Faktoren nicht vollständig vorhersagen, welche schwerverletzten Patienten gute Chancen auf Wiederherstellung und soziale Rehabilitation haben und welche nicht. Einige Studien richteten die Aufmerksamkeit auf den Einfluss psychologischer Faktoren, wie Depressivität und PTBS (posttraumatische Belastungsstörung) [9, 21]. In einigen Arbeiten wurde über die Wichtigkeit sozialer Faktoren, wie den Ausbildungsstand des Patienten, berichtet $[1,14$, 16]. Auch der prätraumatische physische Zustand kann bei der Vorhersage des funktionellen Outcomes von Bedeutung sein. Begleiterkrankungen [10] und BMI (Body-Mass-Index) $[3,17]$ können nicht nur die Überlebensrate der Traumapatienten beeinflussen, sondern auch deren Lebensqualität.

Prognostische Einflüsse können in Relation zum Unfallzeitpunkt in prätraumatische, traumabedingte oder posttraumatische Faktoren eingeteilt werden (• Tab. 1).

Posttraumatische Einflüsse sind für die Rehabilitation besonders interessant, da
Tab. 1 Auswahl von Outcome-Prädiktoren in Relation zum Traumazeitpunkt. (Aus [24])

\section{Prätraumatisch}

Physischer und psychologischer

Zustand (BMI, Begleiterkrankung,

Depressivität)

Demografische Faktoren (Alter, Schweregrad Rehabilitationsdauer

Geschlecht)

Soziale Faktoren (Ausbildungsstand,

Einkommen)

Anzahl der

Verletzungen

Körperliche und psychologische Auswirkungen (Mobilitätseinschränkung, PTBS)

BMI Body-Mass-Index, PTBS posttraumatische Belastungsstörung 
rung der Verweildauer (DRG: „diagnosis related groups") in den unterschiedlichsten Phasen der medizinischen Behandlung und Rehabilitation zur Folge. Dies führt im Weiteren zu einer fehlenden inhaltlichen und zeitlichen Verzahnung der einzelnen Rehabilitationsphasen, und hierdurch bedingt werden berufliche und soziale Folgewirkungen des Unfalls nur unzureichend oder verspätet erkannt. Auch medizinische Fehleinschätzungen und mangelnde Kenntnis des physiologischen Heilverlaufs gehen mit einer zeitverzögerten Durchführung von therapeutischen Interventionen einher. Schließlich resultieren die Mängel in einer sinkenden Motivation des Patienten, der schließlich nicht mehr zur aktiven Mitarbeit bereit ist, die für den Erfolg der Rehabilitation aber unabdingbar ist.

\section{Frührehabilitation}

In den letzten Jahren setzte sich die Erkenntnis durch, dass Rehabilitation frühzeitig, umfassend und als zusammenhängender Vorgang stattfinden muss, damit die Rehabilitationschancen der Patienten optimal genutzt werden können. Mit Inkrafttreten des SGB IX (Sozialgesetzbuch) in Verbindung mit der Ergänzung des $\$ 39$ Absatz 1, SGB V, gehören nun zur „akutstationären Behandlung auch die im Einzelfall erforderlichen und zum frühestmöglichen Zeitpunkt einsetzenden Leistungen zur Frührehabilitation“. Auf dieser gesetzlichen Grundlage wird der Auftrag zur Frührehabilitation nun umgesetzt. Die Frührehabilitation ist in Bezug auf Versorgung, Strukturen, Organisation und Finanzierung so zu gestalten, dass möglichst alle Patienten, bei denen die Notwendigkeit für eine Frührehabilitation besteht, erfasst und optimal versorgt werden [26].

\section{Indikationen}

Das Indikationsspektrum der Frührehabilitation im Akutkrankenhaus ist, was die zugrunde liegenden Krankheitsbilder und -diagnosen angeht, naturgemäß sehr breit. Grund hierfür ist, dass die Aufnahmekriterien aus der Beeinträchtigung der Funktionsfähigkeit begründet sind, und zwar unabhängig von der ICD-Diagno- se (ICD: ,international classification of diseases"). Beispiele für Krankheitsbilder in der unfallchirurgischen Frührehabilitation sind [13]:

- Patienten mit erworbenen Hirnschädigungen, z. B. durch Schädel-HirnTrauma

- Patienten, welche schwere Verletzungen am Stütz- und Bewegungsapparat bzw. an den inneren Organen erlitten haben, z. B. durch Polytrauma

- Patienten mit schwerwiegenden akuten neurologischen Erkrankungen, z. B. Para- und Tetraparese

- Patienten nach Amputationen bei vorliegenden Begleiterkrankungen und erheblicher zusätzlicher Behinderung

- Patienten nach Langzeitbeatmung und Langzeitintensivbehandlung sowie Entwicklung eines CriticalIllness-Syndroms

\section{Abgrenzung zur Rehabilitation}

Die Frührehabilitation beschränkt sich auf die Krankheitsphase, in der noch akutstationärer Behandlungsbedarf besteht, und ist von der postakuten bzw. weiterführenden Rehabilitation abzugrenzen. Das entscheidende Merkmal der Frührehabilitation ist somit die Gleichzeitigkeit der Behandlungsbedürftigkeit im Akutkrankenhaus und der Notwendigkeit rehabilitationsmedizinischer Maßnahmen. Deshalb dürfen Leistungen der Frührehabilitation als Teil der Krankenhausleistungen nur solange erbracht werden, wie eine Krankenhausbehandlung medizinisch notwendig ist. Wenn diese nicht mehr erforderlich ist, erfolgt die ggf. nötige weitere Rehabilitation in einer Rehabilitationseinrichtung.

Frührehabilitation ist nicht gleichzusetzen mit Frühmobilisation. Letztere stellt eine mit geringerem zeitlichem Aufwand erbrachte pflegerische oder therapeutische Maßnahme dar, die der Abwendung, Beseitigung oder Minderung krankheits- und behandlungsbedingter, vorübergehender Immobilisationsfolgen dient. Die Frühmobilisation ist in der Regel auf Einzelmaßnahmen aus dem Therapiebereich beschränkt (z. B. Physiotherapie).
Trauma Berufskrankh 2010 - 12[Suppl 2]: 194-198

DOI 10.1007/s10039-010-1612-0

C) Springer-Verlag 2010

\section{S. Simmel \\ Frührehabilitation nach Polytrauma}

\section{Zusammenfassung}

Die Überlebenschancen Polytraumatisierter haben sich in den letzten Jahrzehnten kontinuierlich verbessert. Es stellt sich daher nicht nur die Frage, ob, sondern wie ein Patient einen schweren Unfall überlebt. Die Kenntnis, welche Parameter das Outcome beeinflussen können, ist entscheidend für die Planung, Organisation und Durchführung einer Rehabilitation nach schweren Verletzungen. Je früher diese beginnt, desto besser ist das Ergebnis. Frührehabilitation ist Teil der Akutbehandlung und erfordert eine enge Verzahnung von Rehabilitationsmedizin und Unfallchirurgie. Der Anspruch an einen optimalen Rehabilitationsprozess stellt hohe Anforderungen an die Klinik und das Rehabilitationsteam, die letztendlich nur von spezialisierten Einrichtungen erfüllt werden können.

\section{Schlüsselwörter}

Frührehabilitation · Polytrauma - Lebensqualität · Outcome · ICF (, ,international classification of functioning, disability and health")

\section{Early rehabilitation after multiple trauma}

\section{Abstract}

The survival chances of multiple trauma patients have risen continuously in recent decades. Therefore, not only the question of whether the patient survives a severe accident arises, but rather how the patient survives it. Knowing which parameters can influence outcome is essential for the planning, organization, and implementation of rehabilitation after a severe injury. The earlier the rehabilitation begins, the better the result. Early rehabilitation is part of acute medical treatment and requires close teamwork between rehabilitation medicine and trauma surgery. Standards for optimal rehabilitation place high demands on hospitals and rehabilitation teams, and can ultimately only be met in specialized facilities.

\section{Keywords}

Early rehabilitation - Multiple trauma - Quality of life - Outcome · ICF (international classification of functioning, disability and health) 
In der postakuten Rehabilitation besteht kein akutmedizinischer Behandlungsbedarf mehr oder zumindest nur in geringem Ausmaß. Voraussetzungen für Leistungen zur medizinischen Rehabilitation sind manifeste, nicht nur vorübergehende Beeinträchtigungen der Aktivitäten und/oder drohende bzw. manifeste Beeinträchtigungen der Teilhabe (Rehabilitationsbedürftigkeit), eine Rehabilitationsfähigkeit und eine positive Rehabilitationsprognose.

\section{Ziele und Inhalte}

Die Rehabilitation sollte von Anfang an integraler Bestandteil der medizinischen Versorgung sein. Sobald als möglich sind der funktionelle Status, das Rehabilitationspotenzial und der Rehabilitationsbedarf des Patienten in die Diagnosestellung einzubeziehen und ein am individuellen Bedarf ausgerichtetes Rehabilitationskonzept in die Krankenbehandlung zu integrieren.

Die fachübergreifende Frührehabilitation ist als multi- und interdisziplinäre, patientenzentrierte Teamarbeit organisiert. Fachübergreifende Frührehabilitation bedeutet dabei, dass Patienten aus verschiedenen medizinischen Fachbereichen, mit zumeist multiplen Schädigungen in unterschiedlichen Organsystemen, behandelt werden. Dabei ergänzen sich 2 unterschiedliche Formen der Leistungserbringung. In Abhängigkeit vom Zustand des Patienten und der Situation in den verschiedenen Akutkrankenhäusern kann die Frührehabilitation sowohl auf eigenständigen bettenführenden Frührehabilitationsabteilungen durchgeführt werden als auch durch mobile Frührehabilitationsteams erfolgen [6].

Der Schwerpunkt der Frührehabilitation liegt zunächst in der Therapie der Körperstrukturen und -funktionen mittels intensiver krankengymnastisch-physikalischer und ergotherapeutischer Behandlung. Daneben sind zur Zielerreichung eine Reihe weiterer Maßnahmen wichtig, die über die rein physische Seite der Beeinträchtigungen hinausgehen. Auf der Grundlage eines bio-psycho-sozialen Modells hebt sich die Frührehabilitation damit deutlich von einer „einfachen“ physiotherapeutischen Weiter- oder Nachbe- handlung ab. Sie hat neben dem Ziel der Wiederherstellung bzw. Verbesserung der körperlichen und mentalen Funktionen sowie der Verhütung von Komplikationen folgende Zwecke und Inhalte:

- Verhinderung von bleibenden Behinderungen und (Schwerst-)Pflegebedürftigkeit

- Frühe Einbindung des Rehabilitationsmediziners bereits in der Akutphase

- Koordination der spezifischen körperlichen und psychosozialen Behandlung zwischen Unfallchirurg und multidisziplinärem Rehabilitationsteam

- Suffiziente psychologische Betreuung des Patienten und seiner Angehörigen

- Einbindung des Patienten in die Behandlung und individuelle Rehabilitationszielsetzung

- Erfassung von rehabilitationsrelevanten Kontextfaktoren und Outcome-Prädiktoren

- Erreichung der „Rehabilitationsfähigkeit“ (z. B. mobil auf Stationsebene, Selbstständigkeit bei den Aktivitäten des täglichen Lebens)

- Verkürzung der Verweildauer im Akuthaus und früher Transfer in spezialisierte Traumarehabilitationszentren zur Optimierung der Rehabilitationsdauer

- Organisation der anschließenden Rehabilitationsphase inklusive Erstellung eines Nachsorgekonzeptes für die postakute stationäre und ambulante Rehabilitationsphase

\section{Mindestmerkmale}

Für eine erfolgreiche Rehabilitation auf der Grundlage eines mehrdimensionalen und multimodalen Rehabilitationskonzeptes ist es von entscheidender Bedeutung, den Patienten zielgerichtet in den Mittelpunkt eines fein abgestimmten Netzwerks von Abklärungen, Maßnahmen und Behandlungen zu stellen. Diese Leistung erfordert ein spezialisiertes Rehabilitationsteam, das finanziert werden muss, und setzt bestimmte Strukturen voraus, die in den OPS-Kodes (OPS: Operationen- und Prozedurenschlüssel, [4]) beschrieben sind.
Die Frührehabilitation wird abgebildet durch:

- die Prozedur 8-559 Fachübergreifende und andere Frührehabilitation,

- die Prozedur 8-552 Neurologischeneurochirurgische Frührehabilitation und

- die Prozedur 8-550 Geriatrische frührehabilitative Komplexbehandlung.

Frührehabilitationsprozeduren, die den jeweiligen Fall zu einer Frührehabilitations-DRG triggern, sind ein systemkonformer Ansatz, Frührehabilitation im DRG-System abzubilden. Aber in vielen DRG sind die erbrachten frührehabilitativen Leistungen nicht erlösrelevant. Auch wird die Dauer der Frührehabilitation erheblich durch die Komplikationen der zugrunde liegenden Akuterkrankung mitbestimmt. Diese bedingen eine unkalkulierbare Varianz der Kosten. Für die Berechnung eigener Fallpauschalen sind die durch das InEK (Institut für das Entgeltsystem im Krankenhaus) erfassten Fallzahlen jedoch zu klein.

Zur Kodierung des OPS-Schlüssels 8-559 „Fachübergreifende und andere Frührehabilitation" sind folgende Mindestmerkmale erforderlich:

- Frührehabilitationsteam unter fachärztlicher Behandlungsleitung (mindestens 5 Jahre in der Rehabilitationsmedizin tätig oder 5 Jahre Tätigkeit in der physikalischen und rehabilitativen Medizin oder Facharzt für physikalische und rehabilitative Medizin)

- Standardisiertes Frührehabilitationsassessment oder Einsatz von krankheitsspezifischen Scoresystemen zur Erfassung und Wertung der funktionellen Defizite in mindestens 5 Bereichen (Bewusstseinslage, Kommunikation, Kognition, Mobilität, Selbsthilfefähigkeit, Verhalten, Emotion) zu Beginn der Behandlung

- Wöchentliche Teambesprechung mit wochenbezogener Dokumentation bisheriger Behandlungsergebnisse und weiterer Behandlungsziele

- Aktivierend-therapeutische Pflege durch besonders geschultes Pflegepersonal (therapeutische Lagerung, Mobilisierung, Körperpflege, Kleiden, Essen und Trinken; Ausscheidungs- 
training, Wahrnehmungsförderung, Aktivierungstherapie, Trachealkanülenmanagement u. a.)

- Vorhandensein von mindestens 4 der folgenden Therapiebereiche: Physiotherapie/Krankengymnastik, Physikalische Therapie, Ergotherapie, Neuropsychologie, Psychotherapie, Logopädie/fazioorale Therapie/Sprachtherapie, künstlerische Therapie (Kunstund Musiktherapie), Dysphagietherapie und Einsatz von mindestens 3 dieser Therapiebereiche in patientenbezogenen unterschiedlichen Kombinationen und unterschiedlichem Zeitaufwand

- Entlassungsassessment zur gezielten Entlassung oder Verlegung des Patienten

\section{Probleme}

Sie bestehen sowohl bezüglich der Durchführung als auch der Finanzierung der Frührehabilitation. Zu Beginn des Rehabilitationsprozesses steht die Problemanalyse, wobei neben einer diagnosebezogenen Betrachtung (ICD-10) die systematische Einbeziehung der ICF (,international classification of functioning, disability and health") äußerst wichtig ist. Dies kann bereits im Bereich der Körperfunktionen und -strukturen bzw. deren Schädigungen von Bedeutung sein: So können 2 Personen mit derselben Verletzung ein unterschiedliches Niveau der Funktionsfähigkeit aufweisen, und 2 Personen mit dem gleichen Niveau der Funktionsfähigkeit haben nicht notwendigerweise das gleiche Verletzungsmuster.

Diese Einbeziehung der ICF erfolgt im DRG-System, das auf ICD-10-Diagnosen und fallgewichtserschwerenden Nebendiagnosen sowie OPS-Prozeduren beruht, nicht. Der Ressourcenverbrauch in der Frührehabilitation wird jedoch mit durch die Krankheitsfolgen (Einschränkungen von Körperstrukturen und -funktionen, Aktivitäten und Partizipation gemäß ICF) bestimmt. Die ICF steht allerdings für den praktischen Einsatz noch nicht zur Verfügung. Deshalb wird im diagnosengetriggerten Fallpauschalensystem derzeit ein großer Teil der nichtgeriatrischen bzw. nicht primär neurologischen Patienten mit Frührehabilitations- bedarf von der Finanzierung der Frührehabilitation ausgeschlossen. Frührehabilitation während des akutstationären Aufenthaltes aber wird in der Regel nur erfolgen, wenn dafür auch eine Finanzierung vorgesehen ist.

Ferner muss ein enormer Aufwand betrieben werden, um die Erfüllung der oben genannten „Mindestmerkmale“ zu dokumentieren. Da die Kodierung der entsprechenden Prozedurenschlüssel zeitabhängig ist, führt dies zu einer aufwändigen Zählung von Therapieminuten. Es kann nicht im Sinne des SGB V sein, dass beispielsweise Patienten mit neurologischen Diagnosen nur dann Frührehabilitation erhalten können, wenn sie 300 min an rehabilitativen Interventionen pro Tag benötigen und erhalten.

Nicht selten ist der Fall, dass zwar keine Akutbehandlung mehr erforderlich, aber der Patient noch nicht rehabilitationsfähig ist. Die Frührehabilitation aber beinhaltet definitionsgemäß nur Rehabilitationsmaßnahmen während der Krankheitsphase, in der ein akutstationärer Behandlungsbedarf besteht. Sie ist von der weiterführenden Rehabilitation abzugrenzen. Die Notwendigkeit der Krankenhausbehandlung wird vom Medizinischen Dienst der Krankenkassen regelmäßig geprüft. Dieses „nicht Fisch, nicht Fleisch“ stellt den Arzt regelmäßig vor die Frage, ob er zu Lasten des Krankenhausträgers oder seines Patienten (be)handeln soll.

$\mathrm{Zu}$ guter Letzt treffen auf der unfallchirurgischen Station gelegentlich 2 Welten aufeinander, der Rehabilitationsmediziner und der Unfallchirurg. Unterschiedliche Behandlungsansätze müssen diskutiert und gemeinsam die beste Lösung für den schwerverletzten Patienten gefunden werden. Dies ist nicht immer einfach und erfordert eine gewisse Toleranz auf beiden Seiten.

\section{Fazit}

Die Frührehabilitation polytraumatisierter Patienten ist ein komplexer Prozess, der parallel und eng verzahnt mit der akutmedizinischen Behandlung abläuft und mit Erreichen der Rehabilitationsfähigkeit endet. Orientiert an der internationalen Klassifikation der Funktionsfähigkeit, Behinderung und Gesund- heit (ICF) müssen neben den Schädigungen der Körperstrukturen und -funktionen auch Störungen der Aktivitäten und der Teilhabe bei der Rehabilitationsplanung berücksichtigt werden. Die genaue Kenntnis möglicher Traumafolgen, nicht nur im physischen, sondern auch im psychischen und sozialen Bereich, sowie der Outcome-Prädiktoren ist für die Steuerung des Rehabilitationsprozesses unabdingbar. Für diese Aufgabe werden multidisziplinäre Teams benötigt, organisiert und geleitet durch einen Rehabilitationsarzt, der Erfahrung im Umgang mit Traumapatienten hat und dem die erforderliche Infrastruktur zur Verfügung steht. Durch die enge Verzahnung von Unfallchirurgie und Rehabilitationsmedizin kann der Rehabilitationsprozess optimiert und verkürzt werden. Aufgrund dieser notwendigen Voraussetzungen ist die Frührehabilitation Schwerstverletzter nur in einem darauf ausgerichteten Traumazentrum sinnvoll.

\section{Korrespondenzadresse}

\section{Dr. S. Simmel}

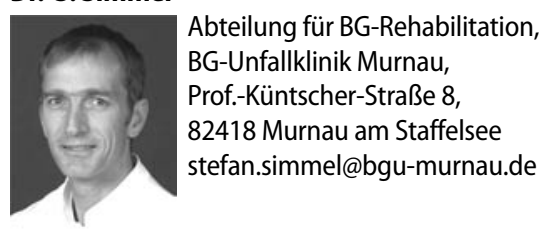

Interessenkonflikt. Der korrespondierende Autor gibt an, dass kein Interessenkonflikt besteht.

\section{Literatur}

1. Anke AGW, Stanghelle JK, Finset A et al (1997) Long-term prevalence of impairments and disabilities after multiple trauma. J Trauma 14:54-61

2. Brennemann FD, Redelmeier DA, Boulanger BR et al (1997) Longterm outcomes in blunt trauma: who goes back to work? J Trauma 42:778-781

3. Byrnes MC, McDaniel MD, Moore MB et al (2005) The effect of obesity on outcomes among injured patients. J Trauma 35:538-542

4. Deutsches Institut für medizinische Dokumentation und Information (DIMDI) (2010) Operationenund Prozedurenschlüssel Version 2010. DIMDI, Köln, www.dimdi.de

5. Fakhry SM, Kercher KW, Rutledge R (1996) Survival, quality of life, and charges in critically ill surgical patients requiring prolonged ICU stays. J Trauma 41:999-1007 
6. Gemeinsame Arbeitsgruppe DRG (gAG-DRG) der Bundesarbeitsgemeinschaft der Akutkrankenhäuser mit Abteilungen für Fachübergreifende Frührehabilitation des Berufsverbandes der Rehabilitationsärzte und der Deutschen Gesellschaft für Physikalische Medizin und Rehabilitation (2009) Positionspapier zur fächerübergreifenden Frührehabilitation. Deutscher Ärzte-Verlag, Köln, aerzteblatt. Insdata.de/download/files/2009/09/down139638. pdf

7. Goins WA, Reynolds HN, Nyanjom D, Dunham CM (1991) Outcome following prolonged ICU stay in multiple trauma patients. Crit Care Med 19:339345

8. Holbrook TL, Hoyt DB (2004) The impact of major trauma: quality-of-life outcomes are worse in women than in men, independent of mechanism and injury severity. J Trauma 56:284-290

9. Holbrook TL, Hoyt DB, Coimbra R et al (2005) Longterm posttraumatic stress disorder persists after major trauma in adolescents: new data on risk factors and functional outcome. J Trauma 58:764-769

10. Holtslag HR, Beeck EF van, Lindeman E, Leenen LP (2007) Determinants of long-term functional consequences after major trauma. J Trauma 62:919927

11. Kinzl L, Gebhard F, Arand M (1996) Polytrauma und Ökonomie. Unfallchirurgie 22:179

12. Knopp W, Kugler J, Reckert P et al (1997) Determinanten der Lebensqualität nach offenem Unterschenkelbruch Typ III: Ergebnisse einer Multicenterstudie. Chirurg 68:1156-1162

13. Leistner K, Stier-Jarmer M, Berleth B et al (2005) Frührehabilitation im Krankenhaus - Definition und Indikation. Phys Med Rehabil Kuror 15:157167

14. MacKenzie EJ, Siegel JH, Shapiro S et al (1988) Functional recovery and medical costs of trauma: an analysis by type and severity of trauma. JTrauma 28:281-297

15. Mahler H, Kulik J (1990) Preferences for health care involvement, perceived control and surgical recovery: a prospective study. Soc Sci Med 31:743-751

16. Meerding WJ, Looman CW, Essink-Bot ML et al (2004) Distribution and determinants of health and work status in a comprehensive population of injury patients. J Trauma 56:150-161

17. Neville AL, Brown CV, Weng J et al (2004) Obesity is an independent risk factor of mortality in severely injured blunt trauma patients. Arch Surg 139:983987

18. Ott R, Holzer U, Spitzenpfeil E, Kastl S et al (1996) Quality of life after survival of severe trauma. Unfallchirurg 99:267

19. Paar O, Kasperk R (1992) Langzeitverlauf nach PoIytrauma. Unfallchirurg 95:78

20. Regel G, Lobenhoffer P, Lehmann U et al (1993) Ergebnisse in der Behandlung Polytraumatisierter Eine vergleichende Analyse von 3406 Fällen zwischen 1972 und 1991. Unfallchirurg 96:350-362

21. Ristner G, Andersson R, Johansson LM et al (2000) Sense of coherence and lack of control in relation to outcome after orthopaedic injuries. Injury 31:751-756

22. Ruchholtz S, Lefering R, Paffrath T et al (2008) Rückgang der Traumaletalität. Dtsch Arztebl 105(13):225-231

23. Scheibler F, Janßen C, Pfaff H (1997) Shared decision making: ein Überblicksartikel über die internationale Forschungsliteratur. Sozialmed Präventivmed 48:11-23

24. Simmel S, Bühren V (2009) Polytrauma überlebt - und was kommt dann? Die Rehabilitation Schwerstverletzter. Unfallchirurg 112:965-974
25. Sittaro NA, Lohse R, Panzica M et al (2007) Hannover-Polytrauma-Langzeit-Studie HPLS (I). Versicherungsmedizin 59:20-25

26. Stucki G, Stier-Jarmer M, Berleth B, Gadomski M (2002) Indikationsübergreifende Frührehabilitation. Phys Med Rehabil Kuror 12:146-156

27. Vazquez Mata G, Rivera Fernandez R, Perez Aragon A et al (1996) Analysis of quality of life in polytraumatized patients two years after discharge from an intensive care unit. J Trauma 41:326-332 